\title{
Esporotricosis granulomatosa: presentación de dos casos inusuales
}

\author{
Max Ramírez-Soto y José Lizárraga-Trujillo
}

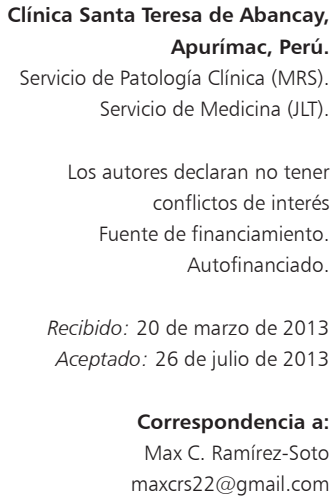

Clínica Santa Teresa de Abancay, Apurímac, Perú. Servicio de Patología Clínica (MRS) Servicio de Medicina (JLT).

Los autores declaran no tener conflictos de interés Fuente de financiamiento. Autofinanciado.

Recibido: 20 de marzo de 2013 Aceptado: 26 de julio de 2013

Correspondencia a: Max C. Ramírez-Soto maxcrs22@gmail.com

\section{Granulomatous sporotrichosis: report of two unusual cases}

Sporotrichosis is a subcutaneous mycosis caused by Sporothrix complex, endemic in Abancay, Peru. Is acquired by traumatic inoculation with plant material. Common clinical presentations are lymphatic cutaneous and fixed cutaneous disease. We report 2 cases of fixed cutaneous sporotrichosis with granulomatous appearance. The first case was a patient of 65 years old with no risk factors and the second case was a 67 year old diabetic patient. Subjects underwent mycological culture with Sabouraud agar, with isolation of Sporothrix schenckii and clinical dignosis of fixed cutaneous sporotrichosis with granulomatous appearance. One patient received oral treatment with saturated solution of potassium iodide (SSKI) with a initial dose of 3 drops tid up to a maximum dose of 40 drops tid. Mycological and clinical cure was achieved after 2 months of treatment. We should consider the unusual clinical presentations of fixed cutaneous sporotrichosis with granulomatous appearance that present morphological and clinical features in diabetic and nondiabetic patients older than 60 years from endemic areas and communicate adequate response to treatment with SSKI in one case.

Key words: Sporotrichosis granulomatous, fixed cutaneous sporotrichosis, Sporothrix schenckii, potassium iodide.

Palabras clave: Esporotricosis granulomatosa, esporotricosis cutánea fija; Sporothrix schenckii, yoduro de potasio.

\section{Introducción}

L a esporotricosis es una micosis subcutánea producida por el complejo dimórfico Sporothrix que incluye cinco especies: S. pallida, S. brasiliensis, S. globosa, S. mexicana y S. schenckii (sensu stricto). Todas estas especies se encuentran ampliamente distribuidas en la naturaleza, especialmente en la tierra, restos vegetales y plantas.

La distribución geográfica de esta micosis es universal, pero predomina en lugares de clima tropical y subtropical: en Sudáfrica, Japón, Francia, en zonas templadas de E.U.A y Canadá. En Centroamérica y en Latinoamérica existen diversos focos endémicos en la zona intertropical en México, Costa Rica, Venezuela, Ecuador, siguiendo por la Cordillera de los Andes, de Perú hasta Bolivia, sur de Brasil, Uruguay y Argentina. En Chile se han presentado casos aislados de esporotricosis humana, confirmándose la presencia de $S$. globosa y $S$. pallida ${ }^{1-3}$. En Perú, se han comunicado numerosos casos en la zona endémica de Abancay, Apurímac (con una incidencia anual de 48-60 casos por 100.000 habitantes $)^{4}$.

La vía de entrada del agente causal es por un traumatismo cutáneo con material vegetal, que se extiende por contigüidad y algunas veces se transmite por rasguño o mordedura de gato $^{1,5}$. Es una enfermedad muy polimorfa, donde las formas clínicas observadas con mayor frecuencia son la cutánea linfática (primo-infección) y la cutánea fija (re-infección), que habitualmente afectan a hospederos sin alteraciones de la inmunidad. Por el contrario, las formas cutáneas superficiales y diseminadas, con o sin compromiso visceral, (osteo-articular, pulmonar y meníngea), son poco frecuentes. Estas últimas afectan a pacientes con alteraciones de la inmunidad adaptativa mediada por células, tales como infección por VIH/SIDA y diabetes mellitus, y suelen desarrollarse cuando es inhalado polvo cargado de esporas del hongo ${ }^{1,6,7}$. De forma excepcional se ha encontrado la forma de lesión cutánea fija asociada a diabetes mellitus tipo $2^{8}$. La presentación clínica depende del sitio de inoculación y de la respuesta inmunológica del hospedero hacia el hongo 9 . El curso clínico puede ser subagudo o crónico y es de fácil control terapéutico con solución saturada de yoduro de potasio (SSKI), de bajo costo y alta efectividad ${ }^{1,10,11}$.

Se presentan dos casos de esporotricosis cutánea fija y se describe el aspecto morfológico granulomatoso de las lesiones, infrecuente de la enfermedad y la eficacia terapéutica con SSKI en la resolución de uno de los casos.

\section{Casos clínicos}

\section{Caso 1}

Mujer de 65 años de edad, originaria y residente de la localidad de Curahuasi, Abancay, Apurímac, Perú. Consultó por presentar una dermatosis localizada en el dorso de la nariz con un tiempo de evolución de 50 días. 
La lesión consistía en una placa no dolorosa de tejido granulomatoso, de bordes bien definidos, eritematosos y presencia de pápulas, sin secreción purulenta, ni exudado sero-hemático (Figuras 1A y B). No presentaba antecedentes familiares patológicos, ni antecedentes personales de diabetes mellitus y/o esporotricosis cutánea. La paciente se dedicaba a las actividades domésticas y manifestó que la lesión apareció después de la picadura de un mosquito. Había recibido tratamiento con clotrimazol al 1\%, sin evolución favorable. De acuerdo a las características clínicas de la lesión, inicialmente se planteó el diagnóstico presuntivo de esporotricosis cutánea fija versus leishmaniosis, por lo que se realizaron exámenes micológicos, parasitológicos, glicemia y hemograma. No fue posible realizar una biopsia de la lesión. El hemograma y la glicemia fueron normales. Al examen microscópico directo con hidróxido de potasio $(\mathrm{KOH})$ al $10 \%$ no se observaron elementos fúngicos. La leishmanina y el frotis con tinción de Giemsa fueron negativos para Leishmania. Para realizar el cultivo micológico se inoculó $0,2 \mathrm{ml}$ de solución salina estéril cargada en una jeringa de
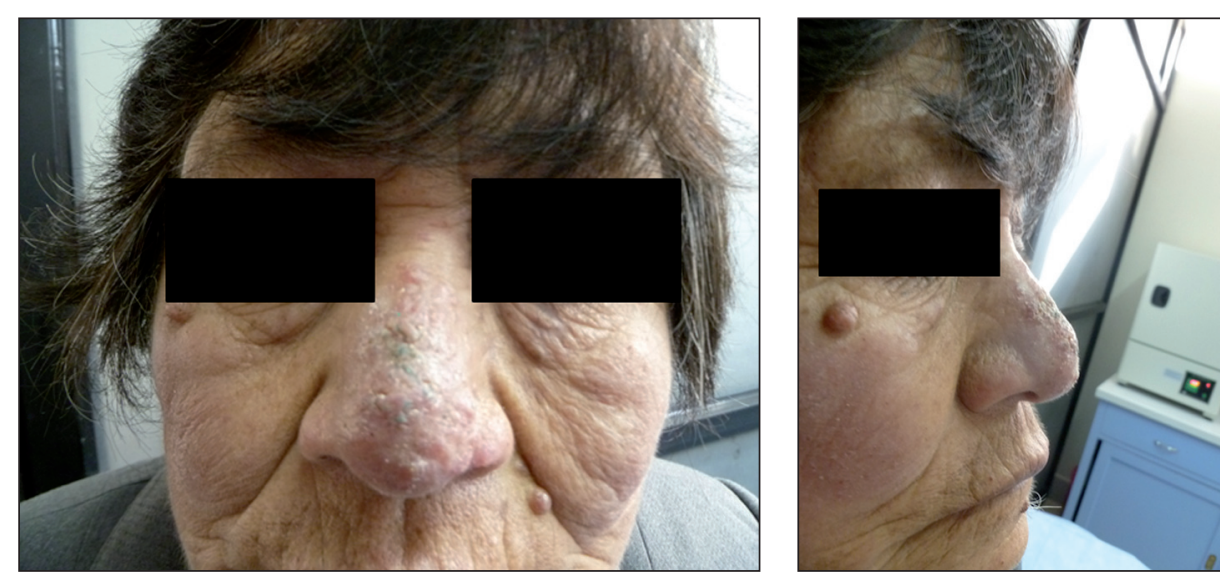

Figura 1. A. Placa de tejido granulomatoso con eritema en el dorso nasal; en la superficie se encuentran pequeñas pápulas. B. Extensión de la lesión del dorso al domus nasal. tuberculina de $1 \mathrm{ml}$ en la lesión y el material aspirado se sembró en agar Sabouraud dextrosa con cloranfenicol a $25^{\circ} \mathrm{C}$. A los ocho días se observó macroscópicamente el desarrollo de colonias blanquecinas, cremosas y finamente radiadas (Figura 2A). En el análisis microscópico con azul de lactofenol, se observaron hifas delgadas y ramificadas acompañados de microconidios compatibles con S. schenckii (Figura 2B). Se confirmó así el diagnóstico de esporotricosis cutánea fija granulomatosa, sin realizar estudios anatomo-patológicos. La paciente no regresó a la consulta, ni al laboratorio para el control clínico de las lesiones, por lo que no recibió tratamiento.

\section{Caso 2}

Mujer de 67 años de edad, agricultora, con antecedente de una diabetes mellitus tipo 2; natural y procedente de la localidad de San Antonio, Tamburco, Abancay, Apurímac, Perú. Consultó por una lesión costrosa melicero-sanguinolenta y granular en la pierna derecha de dos años de evolución. Al examen físico destacaba una lesión cutánea eritemato-violácea de aspecto costroso y

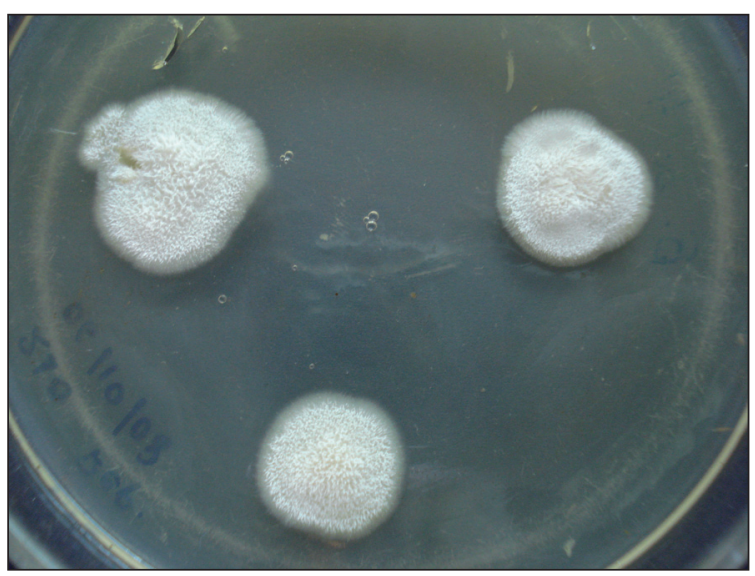

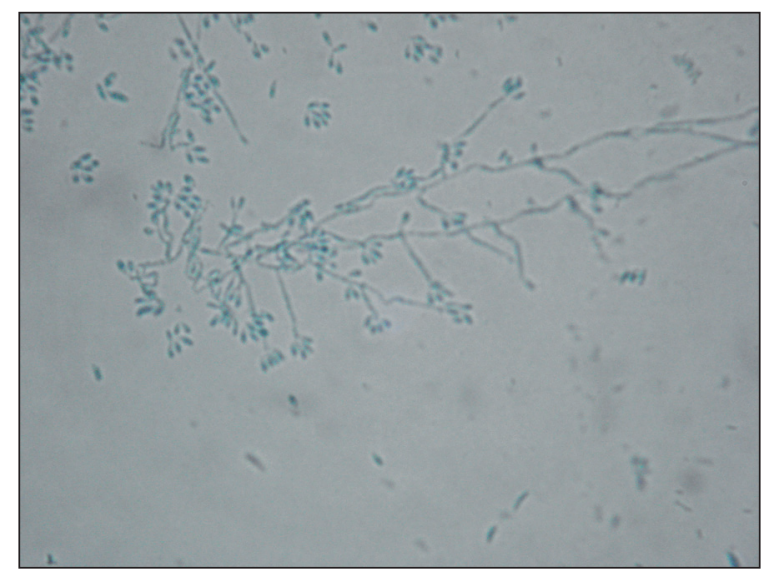

Figura 2. Sporothrix schenckii. A. Cultivo en agar Sabouraud a $25^{\circ} \mathrm{C}$; se observan colonias blanquecinas finamente radiadas. B. Microfotografía a partir de cultivo; se observa hifas delgadas acompañados de microconidios. 
bordes irregulares, de tejido granulomatoso (Figura 3A). A la presión no secretaba material purulento, ni exudado sero-hemático y refería leve dolor. No tenía antecedentes familiares de interés y tampoco evidencia de esporotricosis cutánea previa. Con estos hallazgos se diagnosticó presuntivamente una micosis subcutánea. Entre los exámenes solicitados destacó una glicemia elevada (203 $\mathrm{mg} / \mathrm{dl}$ ) y un hemograma normal. Al examen microscópico directo con $\mathrm{KOH}$ al $10 \%$, no se observaron estructuras micóticas. En este caso, tampoco fue posible realizar el estudio histopatológico de la lesión. Para realizar el cultivo micológico se practicó una punción aspirativa mediante una jeringa de tuberculina cargada con $0,2 \mathrm{ml}$ de solución salina estéril. El material extraído se sembró en agar Sabouraud dextrosa con cloranfenicol a $25^{\circ} \mathrm{C}$. A los 10 días se aislaron colonias cremosas y filamentosas en las que microscópicamente se observaron micelios delgados, acompañados de microconidios sésiles, compatibles con S. schenckii.
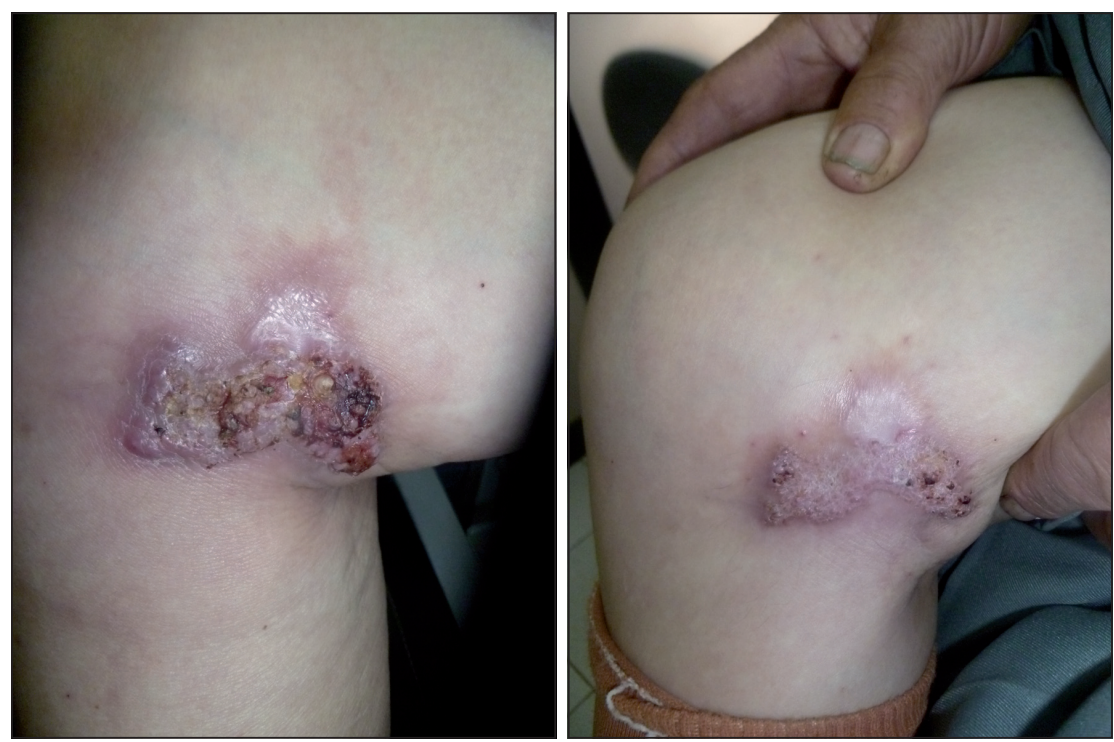

Figura 3.A. Esporotricosis cutánea-fija granulomatosa; lesión eritemato-violácea con granulomas y costras melicéricas. B. Evolución clínica y microbiológica 45 días post-tratamiento.

Tabla 1. Identificación morfo-fisiológica de especies de Sporothrix complex

\begin{tabular}{|c|c|c|c|c|c|}
\hline \multirow[t]{2}{*}{ Especie } & \multirow{2}{*}{$\begin{array}{l}\text { Presencia de } \\
\text { conidios sésiles } \\
\text { pigmentados }\end{array}$} & \multirow{2}{*}{$\begin{array}{l}\text { Colonias en PDA a } \\
30^{\circ} \mathrm{C} \text { exceden los } \\
50 \mathrm{~mm} \text { en } 21 \text { días }\end{array}$} & \multirow{2}{*}{$\begin{array}{c}\text { Crecimiento } \\
\text { a } 37^{\circ} \mathrm{C}\end{array}$} & \multicolumn{2}{|c|}{ Test de asimilación } \\
\hline & & & & Sucrosa & Rafinosa \\
\hline S. pallida & No & Sí & Sí & + & - \\
\hline S. brasiliensis & Sí & No & Sí & - & - \\
\hline S. globosa & Sí & No & No & + & - \\
\hline S. mexicana & Sí & Sí & Sí & + & + \\
\hline S. schenckii & Sí & No & Sí & + & + \\
\hline
\end{tabular}

Marimon, et $\mathrm{al}^{2}$. PDA: agar papa dextrosa.
En base a los antecedentes clínicos y el cultivo, se diagnosticó una esporotricosis cutánea fija granulomatosa. La paciente recibió tratamiento con SSKI vía oral, con una dosis de inicio de tres gotas, tres veces al día hasta llegar a un máximo de 40 gotas, tres veces al día ( 40 gotas $=2 \mathrm{~g}$ de SSKI). A las tres semanas de tratamiento, se había iniciado la cicatrización de la lesión con una negativización micológica. Finalmente se logró la remisión completa de la lesión a los dos meses, quedando una cicatriz residual (Figura 3B).

Prueba del dimorfismo: Para realizar la prueba del diforfismo de $S$. schenckii, el cultivo miceliar en agar Sabouraud dextrosa con cloranfenicol fue subcultivado en agar BHI (infusión cerebro corazón) con $5 \%$ de sangre a $37^{\circ} \mathrm{C}$. A partir del sexto día de incubación se observaron colonias de aspecto levaduriforme. Al examen microscópico con azul de lactofenol se observaron blastoconidios delgados, confirmándose la presencia S. schenckii.

Identificación de la especie: La identificación de las especies de Sporothrix se realizó de acuerdo a la clave morfo-fisiológica de Marimon y cols. ${ }^{2}$ (Tabla 1). Las colonias fueron caracterizadas según color, crecimiento, textura y morfología microscópica (forma y disposición de los conidios) in vitro. El estudio fisiológico se realizó en agar PDA (Difco ${ }^{\mathrm{TM}}$ Laboratorios, BD, REF 213400) a 30,35 y $37^{\circ} \mathrm{C}$ por 21 días, observándose crecimiento a las tres diferentes temperaturas. Las colonias tenían menos de $50 \mathrm{~mm}$ de diámetro hasta los 21 días, de color crema hasta la segunda semana, posteriormente se tornaron oscuras. El perfil de asimilación de carbohidratos (sucrosa y rafinosa) se realizó en una base nitrogenada, observándose positividad para ambos azúcares. En el estudio microscópico se observaron hifas delgadas, hialinas y ramificadas con microconidios sésiles y simpudólicos. La caracterización fenotípica, es decir, el crecimiento a diferentes temperaturas, las características macroscópicas y microscópicas, y la asimilación de los carbohidratos dieron resultados similares a los encontrados para las cepas de referencia de $S$. schenckii informado por Marimon y cols.

La certificación de la especie fue realizada por el Laboratorio de Micología del Centro Nacional de Salud Pública del Instituto Nacional de Salud, Perú (Laboratorio de Referencia Nacional), donde se confirmó la especie S. schenckii.

\section{Discusión}

La esporotricosis es una micosis endémica en las regiones de Perú y México ${ }^{1,4}$. La mayor serie de casos descrita en Perú, se realizó en la Clínica Santa Teresa de Abancay-Apurímac, que es el Centro de Referencia de diagnóstico y tratamiento de esporotricosis. Desde enero de 2004 a diciembre de 2011 se identificaron 485 casos en toda la región de Apurímac, de los cuales 402 fueron de 
la provincia de Abancay. El 63 y 37\% de los casos fueron lesiones cutáneo-linfáticas y fijas, respectivamente ${ }^{12}$. Los dos casos clínicos relatados representan distintas formas de la esporotricosis cutánea fija: un caso asociado a diabetes mellitus tipo $2 \mathrm{y}$ el otro sin evidencia de inmunosupresión, ambos con características morfológicas y clínicas poco habituales. Algunos autores consideran que la forma cutánea fija presenta una variación, la forma cutánea superficial, constituida por placas eritematoescamosas, violáceas y que se manifiesta generalmente en la cara ${ }^{9,13}$. En esta variedad no hay diseminación, la lesión inicial permanece como un nódulo, chancro único o placa escamo-verrucosa con bordes bien limitados ${ }^{14}$. Lavalle y Mariat ${ }^{15}$ consideran que la forma cutánea fija se produce por la reinfección de los pacientes que han desarrollado inmunidad a $S$. schenckii, mientras que la forma linfo-cutánea se manifiesta en pacientes sin contacto previo con el hongo. A diferencia de lo señalado por estos autores, los dos casos aquí presentados no tenían antecedentes de esporotricosis. Además, llama la atención la morfología cutánea fija granulomatosa de las lesiones. Esto probablemente esté relacionado a una inmunidad relativa desarrollada ante una sucesiva exposición al agente etiológico durante sus actividades laborales. A diferencia de la lesión cutánea-linfática que es de fácil diagnóstico, la lesión cutánea fija es de presentación muy polimorfa y puede imitar a otras infecciones cuyos agentes infecciosos pueden originar este síndrome: tales como micobacterias atípicas, nocardiosis, cromomicosis, leishmaniosis cutánea, tularemia, tuberculosis verrugosa e incluso algunos casos de micetoma ${ }^{1}$. En estos casos debe plantearse el diagnóstico diferencial con estas patologías, sobre todo en zonas endémicas.

El primer caso correspondió a una lesión cutánea fija de aspecto granulomatoso en la nariz relacionada con la picadura de un mosquito. En la literatura científica existen muy pocos casos con esta localización anatómica, siendo más frecuente en las extremidades ${ }^{16}$. No se ha descrito la forma granulomatosa en lesiones cutáneo fijas. El antecedente de picadura de un mosquito nos hizo pensar primero en leishmaniosis cutánea por la forma clínica de la lesión. Precisamente, el principal diagnóstico diferencial de la esporotricosis cutánea fija, es la leishmaniosis cutánea $^{1,17}$. Por otro lado, la presencia de eritema y pápulas en la lesión nos llevó a considerar la esporotricosis como uno de los diagnósticos más probables.

El segundo caso correspondió a una paciente con esporotricosis cutánea fija granulomatosa asociada a diabetes mellitus tipo 2, con dos años de evolución. Los pacientes diabéticos tienen alteraciones celulares y humorales que los predisponen a procesos infecciosos de diversa índole ${ }^{18,19}$. Algunas formas de esporotricosis, como la cutánea diseminada o cutánea-hematógena, se presentan habitualmente en pacientes con estados patológicos de inmunosupresión que afectan la inmunidad celular y humoral, como la diabetes mellitus tipo 2, la infección por VIH/SIDA, neoplasias hematológicas y algunos otros estados de inmunosupresión parcial, como la desnutrición y el alcoholismo crónico. En cambio, es poco habitual encontrar la forma cutánea fija en pacientes diabéticos o inmunocomprometidos ${ }^{1,6,8}$. Otros autores consideran que la forma clínica cutánea fija está vinculada a la inmunidad desarrollada ante la primera infección ocurrida años atrás ${ }^{20}$; sin embargo, nuestra paciente no tenía evidencia de esporotricosis cutánea previa. Estas consideraciones nos hacen pensar que posiblemente la incidencia de esporotricosis podría estar aumentando en los pacientes inmunocomprometidos, y por tanto, debe establecerse el diagnóstico diferencial con otras infecciones.

El estándar de oro para el diagnóstico de la esporotricosis es el cultivo micológico. Otras técnicas complementarias son la histopatología, el examen directo y la intradermo-reacción ${ }^{1,11,12,17}$. Estudios recientes han demostrado que $S$. schenckii es un complejo de al menos seis especies filogenéticas, algunas de las cuales predominan en ciertas regiones geográficas. Estas mismas investigaciones han permitido asociar, mediante características fenotípicas y moleculares, nuevas especies de Sporothrix (S. brasiliensis, S. mexicana) con infecciones humanas, las que se encuentran exclusivamente en Brasil y México, respectivamente. En cambio S. schenckii y S. globosa tienen distribución trans-oceánica ${ }^{2,21}$. Los estudios micológicos de aislamiento e identificación de S. schenckii en los dos casos presentados son, a nuestro parecer, de gran importancia clínica. En las últimas décadas, se ha descrito que el mayor número de casos de esporotricosis asociados a grandes epidemias, debido a transmisión zoonótica, estuvieron asociados a S. schenckii y $S$. brasiliensis. Las manifestaciones clínicas que ocasionan estas especies son lesiones cutáneas fijas, linfáticas y diseminadas. En cambio, S. globosa no provocaría infección cutánea o diseminada ${ }^{2,22,23}$ ya que no logra un adecuado desarrollo a $37^{\circ} \mathrm{C}$; sin embargo, la especie $S$. globosa es de importancia médica y se ha asociado a casos de esporotricosis linfo-cutánea ${ }^{2,3}$. Con estos antecedentes podemos concluir que las especies de Sporothrix difieren en su potencial patogénico, donde $S$. schenckii además de ocasionar lesiones linfo-cutáneas y fijas, puede generar lesiones granulomatosas dependiendo del estado inmune del hospedero.

Según las guías de la Infectious Diseases Society of America el tratamiento de elección para la esporotricosis cutánea fija y linfática en adultos es itraconazol vía oral, $200 \mathrm{mg}$ al día, por un total de tres a seis meses. También puede usarse SSKI en dosis crecientes de cuatro hasta 50 gotas, tres veces al día, después de las comidas para permitir una mejor tolerancia ${ }^{10,11}$. La anfotericina B se reserva para las formas diseminadas o sistémicas. Otros 
tratamientos descritos incluyen: termoterapia (compresas calientes a $42^{\circ}$ ó $43^{\circ}$ durante 40 a 60 min por día), fluconazol, terbinafina, cotrimoxazol y griseofulvina ${ }^{11,24}$. En nuestra experiencia, el esquema de tratamiento estandarizado con SSKI por vía oral, en dosis crecientes de cuatro hasta 40 gotas, tres veces al día, ha mostrado resultados favorables en los pacientes adultos ${ }^{12}$. En el segundo caso, la paciente presentó una buena respuesta al tratamiento, sin ningún evento adverso. Sin embargo, se debe tener presente los posibles efectos colaterales: iododerma, alteraciones digestivas, supresión tiroidea, hipertrofia de las glándulas parótidas y lacrimales, entre otras. El mecanismo de acción de este compuesto es desconocido, ya que no es fungistático ni fungicida. Se cree que influye en la reacción inmunitaria del hospedero contra el microorganismo. Por tanto, sugerimos que el tratamiento con SSKI puede usarse en pacientes diabéticos, por ser bien tolerado, si se administra adecuadamente.

Estudios recientes evaluaron el perfil de susceptibilidad in vitro a los agentes antifúngicos para determinar la mejor opción terapéutica para cada caso de esporotricosis, y concluyeron que terbinafina fue el fármaco más activo contra S. schenckii, seguido de ketoconazol, itraconazol $\mathrm{y}$ anfotericina $\mathrm{B}$, mientras que fluconazol y caspofungina fueron menos activos. En los aislados de S. schenckii y $S$. brasiliensis de esporotricosis humana no se encontró resistencia a itraconazol ${ }^{23,25,26}$; mientras que en algunas cepas de $S$. pallida y $S$. luriei, aisladas de esporotricosis animal (gatos y perros), se describió resistencia a itraconazol y resistencia cruzada a los otros azoles ${ }^{23}$.

Debido a que actualmente se han descrito nuevas especies de Sporothrix en el mundo, es importante que en
Perú se desarrollen estudios de epidemiología molecular para distinguir las variantes de Sporothrix, así como sus respectivos patrones de susceptibilidad antifúngica. La esporotricosis ocurre frecuentemente en muchos lugares de Perú y de Latinoamérica, sobre todo en áreas rurales; de ahí que sea poco conocida por los médicos que trabajan en zonas urbanas. Por este motivo, nos parece importante presentar estos dos casos para dar a conocer las manifestaciones clínicas de casos inusuales de esporotricosis, como lo es la forma cutánea fija granulomatosa.

\section{Resumen}

La esporotricosis es una micosis subcutánea ocasionada por el complejo Sporothrix, endémica en Abancay, Perú. Se adquiere por inoculación traumática con material vegetal. Las formas clínicas comúnmente descritas son la cutánea linfática y cutánea fija. Presentamos dos casos de pacientes adultos con una esporotricosis cutánea fija granulomatosa. El primer caso era una paciente de 65 años de edad, sin antecedentes de riesgo y el segundo era una paciente de 67 años, diabética. El diagnóstico se realizó por cultivo micológico aislándose Sporothrix schenckii. Un caso se trató con solución saturada de yoduro de potasio (SSKI), vía oral, alcanzando la cura micológica y clínica tras dos meses de tratamiento. La otra paciente no acudió a tratamiento. Se revisan las presentaciones clínicas inusuales de la esporotricosis cutánea fija y la forma granulomatosa que presenta características morfológicas y clínicas poco habituales en pacientes diabéticos y no diabéticos mayores de 60 años provenientes de zonas endémicas.

\section{Referencias bibliográficas}

1.- Arenas R. Micología Médica Ilustrada. $3^{\mathrm{a}}$ ed. McGraw-Hill Interamericana 2008; p. 149-59.

2.- Marimon R, Cano J, Gené J, Sutton D A, Kawasaki M, Guarro J. Sporothrix brasiliensis, S. globosa, and S. mexicana, three new Sporothrix species of clinical interest. J Clin Microbiol 2007; 45: 3198-206.

3.- Cruz R, Vieille P, Oschilewski D. Aislamiento ambiental de Sporothrix globosa en relación a un caso de esporotricosis linfo-cutánea. Rev Chilena Infectol 2012; 29: 401-5.

4.- Papas P G, Tellez I, Deep A E, Nolasco D, Holgado W, Bustamante B. Sporotrichosis in Peru: description of an area of hyperendemicity. Clin Infect Dis 2000; 30: 65-70.

5.- de Lima Barros M B, de Oliveira Schubach A, Galhardo M C, Schubach T M, dos Reis R $\mathrm{S}$, Conceicao M J, et al. Sporotrichosis with widespread cutaneous lesions: report of 24 cases related to transmission by domestic cats in Rio de Janeiro, Brazil. Int J Dermatol 2003; 42: 677-81.

6.- Shaw J C, Levinson W, Montanaro A. Sporotrichosis in the acquired immunodeficiency syndrome. J Am Acad Dermatol 1989; 21: 1145-7.

7.- Vega-Morquecho O, Bonifaz A, BlancasGonzález F, Mercadillo-Pérez P. Esporotricosis cutáneo-hematógena. Rev Med Hosp Gen Mex 2002; 65: 98-101.

8.- Motswaledi H, Nkosi L, Moloabi C, Ngobeni K, Nemutavhanani D, Maloba B. Sporotrichosis: A case report and literature review. J Clin Exp Dermatol Res 2011; 2: 132. doi:10.4172/2155-9554.1000132.

9.- Bonifaz A, Vázquez-González D. Sporotrichosis: an update. G Ital Dermatol Venereol 2010; 145: 659-73.

10.- Cabezas C, Bustamante B, Holgado W, Begue R. Treatment of cutaneous sporotrichosis with one daily of potassium iodide. Pediatr Infect Dis J 1996; 15: 352-4.
11.- Kauffman C A, Bustamante B, Chapman S W, Pappas P G. Clinical practice guidelines for the management of sporotrichosis: 2007 update by the Infectious Diseases Society of America. Clin Infect Dis 2007; 45: 1255-65.

12.- Ramírez-Soto M, Lizarraga-Trujillo J, Ticona-Sánchez E, Carrión-León O, Borda-López S. Perfil clínico-epidemiológico de esporotricosis en una clínica de referencia en Abancay, Perú: 2004-2011. Rev Peru Epidemiol 2012; 16: 121-26.

13.- Bonifaz A, Saúl A, Montes de Oca G, Mercadillo P. Superficial cutaneous sporotrichosis in specific anergic case. Int $\mathrm{J}$ Dermatol 1999; 38: 700-3.

14.- Villaca-Neto C M, Rossetti R B, Fischman O, Paschoal L H. Localized cutaneous verrucous sporotrichosis of 26 years duration. Mycoses 1988; 31: 353-5.

15.- Lavalle P, Mariat F. Sporotrichosis. Bull Inst Pasteur 1983; 81: 295-322.

16.- Rubio G, Sánchez G, Porras L, Alvarado Z. 
Esporotricosis: prevalencia, perfil clínico y epidemiológico en un centro de referencia en Colombia. Rev Iberoam Micol 2010; 27: 75-9.

17.- de Lima Barros M B, Schubach A, Francesconido-Valle A C, Gutiérrez-Galhardo M C, Schubach T M, Conceição-Silva F, et al. Positive Montenegro skin test among patients with sporotrichosis in Rio De Janeiro. Acta Trop 2005; 93: 41-7.

18.- Fiad M E, Quenardelle A S, Giménez M F. Esporotricosis. Act Terap Dermatol 2001; 24: 324-31.

19.- Méndez-Tovar L J, Anides-Fonseca A E, Peña González G, Manzano-Gayosso P, López-Martínez R, Hernández-Hernández F, et al. Esporotricosis cutánea fija incógnita. Rev Iberoam Micol 2004; 21: 150-2.

20.- Starck F, Saponaro A E, Marini M A,
Casas J G, Vigovich F, Agorio I. Esporotricosis cutánea fija. A propósito de un caso. Arch Argent Dermatol 2011; 61: 8-11.

21.- Marimon R, Gené J, Cano J, Trilles L, Dos Santos Lazéra M, Guarro J. Molecular phylogeny of Sporothrix schenckii. J Clin Microbiol 2006; 44: 3251-6.

22.- Rodrígues A M, De Melo Teixeira M, De Hoog G S, Schubach T M, Pereira S A, Fernandes G F, et al. Phylogenetic analysis reveals a high prevalence of Sporothrix brasiliensis in feline sporotrichosis outbreaks. PLoS Negl Trop Dis 2013; 7: e2281.

23.- Oliveira D C, Lopes P G, Spader T B, Mahl C D, Tronco-Alves G R, Lara V M, et al. Antifungal susceptibilities of Sporothrix albicans, S. brasiliensis, and $S$. luriei of the $S$. schenckii complex identified in Brazil.
J Clin Microbiol 2011, 49: 3047-9.

24.- Haruna K, Shiraki Y, Hiruma M, Ikeda S, Kawasaki M. A case of lymphangitic sporotrichosis occuring on both forearms with a published work review of cases of bilateral sporotrichosis in Japan. J Dermatol 2006; 33: 364-7.

25.- Marimon R, Serena C, Gené J, Cano J, Guarro J. In vitro antifungal susceptibilities of five species of Sporothrix. Antimicrob Agents Chemother 2008; 52: 732-4.

26.- Silveira C P, Torres-Rodríguez J M, Alvarado-Ramírez E, Murciano-Gonzalo F, Dolande M, Panizo M, et al. MICs and minimum fungicidal concentrations of amphotericin B, itraconazole, posaconazole and terbinafine in Sporothrix schenckii. J Med Microbiol 2009; 58: 1607-10. 